\title{
White versus gray matter function as seen on neuropsychological testing following bone marrow transplant for acute leukemia in childhood
}

\author{
Fiona S Anderson' \\ Alicia S Kunin-Batson' \\ Joanna L Perkins ${ }^{2}$ \\ K Scott Baker ${ }^{3}$ \\ 'Divisions of Pediatric Clinical \\ Neuroscience; ${ }^{2}$ Department of \\ Pediatric Hematology/Oncology, \\ Children's Hospitals and Clinics, \\ Minneapolis, MN, USA and \\ ${ }^{3}$ Hematology/Oncology/BMT, \\ Department of Pediatrics, University \\ of Minnesota, Minneapolis, MN, USA
}

Correspondence: Fiona S Anderson Division of Pediatric Clinical

Neuroscience, Mayo Mail Code 486, 420 Delaware Street Southeast, Minneapolis, MN 55455, USA

Tel +16126249110

Fax + $612624768 \mid$

Email willi040@umn.edu

\begin{abstract}
Current theory suggests that neurocognitive late effects of treatments for childhood cancer such as difficulties with attention, processing speed and visual-motor ability are the result of white matter damage. Neuroimaging studies have produced a variety of white matter findings. However, although white matter is thought to be differentially affected, previous studies have not demonstrated a discrepancy between white and gray matter function. The present study included 36 children treated for childhood leukemia with hematopoietic stem cell transplant (HCT). Their performance on neurocognitive measures traditionally thought to measure white matter was compared to performance on measures thought to measure gray matter function. Composite white and gray matter standard scores were created based on neuropsychological measures that individuals with known white or gray matter damage perform poorly. As predicted, composite white matter scores $($ mean $=98.1)$ were significantly lower $(t=2.26, p=0.03)$ than composite gray matter scores $($ mean $=102.5)$. Additionally, as gray matter performance increased, the difference between gray and white matter scores increased $(\mathrm{R}=0.353, \mathrm{p}=0.035)$. Overall, the results of this study support the current theory that white matter damage is responsible for the more subtle neurocognitive late effects resulting from treatment for childhood leukemia.
\end{abstract}

Keywords: late effects of cancer treatment, leukemia, neuropsychology, white matter, brain function

\section{Introduction}

Childhood leukemia survivors are a population with well-documented neurocognitive late effects. Chemotherapy and radiation both are well-documented causes of declines in neurocognitive functioning (Waber 2000; Mulhern 2001; Butler and Mulhern 2005; Butler and Haser 2006). With continuing advances in therapies, these neurocognitive late effects tend to be domain specific and relatively mild, with overall cognitive functioning remaining within the average range. With current treatment regimens, there is generally no significant cognitive impairment even in bone marrow transplant survivors (Phipps et al 1995; Perkins et al 2007) who have undergone not only the initial treatment for their cancer, but the chemotherapy and sometimes radiation given as the preparative regimen for transplant. However, specific neurocognitive domains like attention, processing speed, and visual-motor precision continue to emerge as domains affected (if not impaired) by chemotherapy alone (Waber et al 2000; Butler and Haser 2006) as well as radiation (Mulhern 2001; Butler and Haser 2006) in leukemia survivors. Patients who have survived bone marrow transplant as treatment for childhood leukemia are at particular risk for neurocognitive late effects, given the cumulative effects on the central nervous system of the chemotherapy and often radiation treatments they have undergone (Leiper 2002). 
The most widely accepted current theory for the known neurocognitive effects of cancer treatment is damage to the white matter in the brain (Butler and Haser 2006; Reddick et al 2006). The goal of this study is to examine this current theory by comparing performance on tests traditionally thought to measure white versus gray matter function in a population of children treated for childhood leukemia with hematopoietic stem cell transplant. Patients are expected to perform worse on tests of white matter function than on tests of gray matter function.

\section{Theoretical models for neurocognitive changes}

Axons conveying information from one neuron to the next within the brain are insulated by myelin, which is what gives white matter its white appearance. Functionally, the connectivity between different brain regions is thought to be evident in neuropsychological measures such as processing speed and visual-spatial or visual-motor tasks that depend on communication between multiple regions of the brain (Kolb and Wishaw 2003). Reddick and colleagues (2006) suggest that "the developing brain may be more susceptible to damage because newly synthesized myelin has higher metabolic activity and lower stability that make it more vulnerable to the toxic effects of therapy." Mulhern and colleagues (Mulhern 1999; Reddick 2000; Mulhern 2001; Reddick 2006) demonstrated a correlation between failure to develop or loss of normal appearing white matter, cranial radiation therapy, and IQ in pediatric brain tumor survivors.

Although cranial radiation therapy has been strongly implicated in white matter changes, chemotherapy alone is also thought to have similar effects (Butler and Mulhern 2005; Moore 2005). A very recent study has linked white matter volume with neurocognitive impairments in childhood ALL (Reddick et al 2006). Another recent study has linked fractional anisotropy in white matter to IQ in childhood cancer survivors (Khong et al 2006) Other diseases also support this model. For example, children with childhoodonset cerebral adrenoleukodystrophy, an X-linked disorder of the peroxisomes that is characterized by elevations in very-long-chain fatty acids, have significant white matter disease. The extent of white matter disease correlates with their performance on visual-perceptual tasks including Performance IQ and Judgment of Line Orientation and on sustained attention measures (Shapiro 2000). White matter lesions in an elderly population were associated with slower psychomotor speed (de Groot and Hofman 2000). And white matter disease is also thought to be responsible for subtle deficits in psychomotor efficiency in diabetes (Ryan 2003).

It is difficult to separate gray and white matter tests, as few tasks can truly be localized to one area of the brain. However, gray matter function can be assessed through more language-based reasoning and memory measures. Language is generally considered a cortical function, usually in the left hemisphere, and the deep gray matter of the hippocampus is also known to be involved in verbal memory (Lezak 1995). Temporal lobe epilepsy research has demonstrated an association between deficits in these functions in patients with known cortical abnormalities (Bennett and Ho 1989). And patients with known white matter lesions were found to be unaffected on verbal list-learning tasks (de Groot and Hofman 2000).

\section{Neuroradiologic findings}

Evidence of small hemorrhages in the white matter have been found in a greater proportion of leukemia survivors, which has been attributed to neurotoxicity of methotrexate (Paako et al 2003) and to radiation (Chan et al 2006). Wilson and colleagues (1991) showed structural white matter changes on MRI during chemotherapy for ALL, but the changes were no longer evident at three year follow-up. Neuroimaging techniques have advanced in recent years from basic structural MRI to more sophisticated and sensitive techniques such as volumetrics, diffusion tensor imaging, and magnetic resonance spectroscopy. Gathering evidence from these more advanced techniques suggests that in addition to the previously identified hemorrhagic lesions, there is diffuse lasting white matter damage that is likely attributable to swelling (Haykin et al 2006). Preliminary diffusion tensor imaging studies have demonstrated decreased white matter diffusion anisotropy after chemotherapy and cranial radiation for childhood medulloblastoma and leukemia, which also correlated with IQ (Leung 2004; Khong et al 2006). Magnetic resonance spectroscopy studies have shown a reduction in $\mathrm{N}$-acetyl-aspartate ratios in white matter of leukemia survivors (Chan et al 2001; Chu et al 2003).

Despite the evidence supporting the theory that white matter is differentially affected and is responsible for neurocognitive changes following cancer treatment, it remains a relatively untested hypothesis. No research has specifically compared gray and white matter structure or function in childhood leukemia and/or bone marrow transplant survivors in conjunction with neurocognitive functioning. As survivorship increases, the importance of understanding how cancer treatment affects a child's brain and its functioning is 
greater than ever before. Studies that provide a link between the physiological effects on the brain of cancer treatments and functional neurocognitive outcomes that can affect quality of life can potentially inform treatment decisions at several levels. They can help pediatric oncologists choose the safest and most effective treatment for children, monitor treatment effects, and help inform the educational plan for children as their cognitive strengths and weaknesses and the reasons for these are better understood. In addition, through this kind of research, it may eventually be possible to use new, non-invasive neuroimaging techniques such as diffusion tensor imaging for early identification of subtle white matter changes that may forecast varying degrees of neurocognitive late effects.

\section{Methods}

\section{Participants}

Eligible subjects were identified from the University of Minnesota HCT database with a history of ALL or AML, who had survived $\geq 1$ year after $\mathrm{HCT}$, and were currently in remission. Eligible patients were initially mailed a letter describing the study. In those cases without response, an attempt was made to contact patients by telephone. Data was available from a total of 36 patients on all necessary measures.

Participants ranged from 8 to 18 years of at testing. They were transplanted between 6 months-old and 12 years-old (mean of 6 years-old) between 1981 and 2000. One patient received two transplants. They were anywhere from 1 year to $14 \frac{1}{2}$ years from time of transplant at testing (mean of 7 years). Thirty-two patients had received total body irradiation while 4 had not. All received chemotherapy on a variety of different protocols. Children with a diagnosis of Down syndrome were not included in this study.

\section{Data collection}

The HCT database of the University of Minnesota Blood and Marrow Transplantation Program was utilized for obtaining relevant transplant related data specific transplant related outcomes. After obtaining informed consent, survivors (accompanied by a parent) were scheduled through the General Clinical Research Center at the University of Minnesota. A description of the complete research protocol can be found in Perkins and colleagues (2007) Long-term Follow-up of Children who Underwent Hematopoeitic Cell Transplant (HCT) for AML or ALL at Less than Three Years of Age: The University of Minnesota Experience. All participants underwent the same set of neuropsychological tests, administered by a psychometrist who was blinded to the patients' treatment history. Scoring and analysis of these tests is also standardized and was performed by the same individual. All components of this testing were standardized, age-specific and are utilized in routine clinical neuropsychological practice. Testing was completed in the neuropsychology clinic at The University of Minnesota Hospital and in the GCRC.

\section{Composite variables}

Composite variables were formed using a disease model by averaging standard scores across specific measures on which people with known white or gray matter involvement do poorly. All scores were transformed to standard scores. Verbal reasoning and memory are thought to reflect gray matter functions, while attention, processing speed, and visual-spatial and visual-motor coordination are thought to reflect white motor functioning. A gray matter composite was calculated as a mean of the total score for trials 1-5 on the California Verbal Learning Test - Children's version (CVLT-C) and the Verbal Intellectual Quotient (VIQ) on the Wechsler Abbreviated Scale of Intelligence (WASI). The white matter composite score was calculated as an average of a Conners' Continuous Performance Test (CPT) composite (an average of the omissions, response time and variability scores, created so as to not weight one test more heavily than the other) and the Block Design score from the WASI.

\section{Analyses}

Descriptive statistics were run on the variables making up the composite gray and white matter scores, and also on the gray and white matter composite variables. A paired sample, two-tailed t-test was run to test for differences between performance on gray versus white matter tests. Finally a Pearson correlation coefficient was computed for overall gray matter score and the difference between scores on gray and white matter measures.

\section{Results}

Overall, results of descriptive statistics are consistent with previous literature in finding that intellectual ability remained comparable to the normative population (mean $=100$, standard deviation $=15$ ). Verbal IQ was slightly higher than Performance IQ, but not significantly so, and the standard deviation of these scores was not significantly different from normal. CPT scores also fell within the average range, but had a wide range. Subjects also scored similar to the average population on the CVLT-C. Table 1 summarizes these descriptive statistics. 
Table I Descriptive statistics

\begin{tabular}{lllll}
\hline & Minimum & Maximum & Mean & Standard deviation \\
\hline WASI Full scale IQ & 74 & 127 & 101.53 & 14.68 \\
WASI Verbal IQ & 71 & 140 & 100.90 & 14.46 \\
CPT Omissions standard score & 40 & 179 & 105.34 & 24.57 \\
WASI Block design & 67 & 127 & 97.50 & 17.99 \\
CPT Response time standard score & 28 & 151 & 93.88 & 24.03 \\
CPTVariability standard score & 40 & 136 & 97.54 & 22.34 \\
CVLT Trials I-5 standard score & 76 & 124 & 103.33 & 11.59 \\
\hline
\end{tabular}

Abbreviations: CPT, Conner's Continuous Performance Test; CVLT, California Verbal Learning Test; WASI,Wechsler Abbreviated Scale of Intelligence.

The means and standard deviations of the white and gray matter composite scores also fell within the average range (Table 2). Although within the average range, however, there was a significant difference between gray and white matter function $(\mathrm{t}=2.26, \mathrm{p}=0.03)$, with subjects scoring lower on the white matter composite than on the gray matter composite (Table 3).

Upon completion of testing, it was apparent from qualitative review of the data that while there were quite a few patients who performed well above average on most tasks, there were also several who were globally impaired. It seemed that some patients were globally impaired, reflecting damage to their whole brains, while patients with only subtle difficulties evidenced those difficulties on the white matter tests. To address this question, a Pearson correlation coefficient was calculated for the difference between scores on gray and white matter measures and gray matter performance. As gray matter performance increased, the difference between gray and white matter scores increased $(R=0.353, p=0.035)$. That is, subjects who performed poorly on gray matter tests, performed similarly badly on white matter tests. However, patients who scored closer to average or above average had a greater discrepancy between their gray and white matter test scores.

\section{Discussion}

Overall, the results of this study support previous research documenting only minimal neurocognitive sequelae for most transplant survivors, but greater effects on tasks thought to reflect white matter function than on tasks thought to measure gray matter function. That is, the chemotherapy and radiation associated with stem cell transplant and general treatment for childhood leukemia appears to differentially affect

Table 2 Paired samples statistics

\begin{tabular}{lll}
\hline & Mean & Standard deviation \\
\hline White & 98.07 & 13.06 \\
Gray & 102.53 & 11.28 \\
\hline
\end{tabular}

white matter over gray matter in the brain. Additionally, the discrepancy between performance on gray versus white matter tasks increased as overall level of functioning increased. Although some patients experience CNS toxicity that leads to general cognitive compromise, those with only subtle difficulties evidence those difficulties differentially on white matter tests.

These findings lend support to the current hypothesis that white matter damage is responsible for most neurocognitive late effects. Previous studies have shown transient hyperintensities in white matter. Reddick and colleagues (Reddick 2006) suggested that these transient hyperintensities observed on MRI may lead to permanent subtle white matter damage, as seen in the persistent white matter volume changes and associated functional declines following treatment for childhood leukemia.

The question becomes how and when we can best identify these subtle white matter changes. As therapies continue to improve, quality of life and even minor declines in functioning become increasingly important. Identifying even the most subtle effects of different chemotherapies will be helpful to both patients and researchers. The earlier the identification of even subtle brain damage, the easier it will be for patients to develop compensatory strategies and effectively reintegrate into the classroom. Also, the ability to identify subtle changes as early as possible will also help researchers as they develop increasingly effective therapies with fewer and fewer side effects. Newer neuroimaging techniques such as diffusion tensor imaging (DTI) paired with domain-specific neurocognitive testing may help in the early identification of this subtle white matter damage.

The current study had several limitations. First, without baseline data or a matched control sample, it was impossible to determine how subjects' performance compared with their functioning prior to their illness or with similar individuals without leukemia or its treatments. Additionally, this was a very heterogeneous population, including diagnoses of AML and ALL, with differing treatments (including many 
Table 3 Paired samples test

\begin{tabular}{|c|c|c|c|c|c|c|}
\hline \multicolumn{5}{|c|}{ Paired differences } & \multirow[t]{2}{*}{$\mathbf{t}$} & \multirow[t]{2}{*}{ Sig. (2-tailed) } \\
\hline \multirow[t]{3}{*}{ Mean } & \multirow{3}{*}{$\begin{array}{l}\text { Standard } \\
\text { deviation }\end{array}$} & \multirow[t]{3}{*}{ SEM } & \multirow{2}{*}{\multicolumn{2}{|c|}{$\begin{array}{l}95 \% \text { Confidence } \\
\text { interval of the difference }\end{array}$}} & & \\
\hline & & & & & & \\
\hline & & & Lower & Upper & & \\
\hline 4.49 & II.89 & 1.98 & 0.46 & 8.51 & 2.26 & 0.03 \\
\hline
\end{tabular}

Abbreviation: SEM, standard error of mean.

with radiation, a few without). Time since diagnosis and treatment was variable, as was age at diagnosis and treatment. These are all variables known to play a role in the emergence of neurocognitive late effects. Additionally, although the composite variables were developed based on prior studies showing that known lesions in white or gray matter differentially affect performance on those tests, this is a less than perfect method. Real world functional tasks generally activate a number of regions or circuits within the brain (Page 2006), and research has not combined functional magnetic resonance imaging with functional activities to develop specific gray or white matter tasks.

However, the fact that results reached statistical significance even with these limitations suggests that the white matter theory is robust, and worthy of further exploration. Future studies should utilize the most sensitive neuroimaging and neuropsychological techniques available to refine our understanding of how white matter is affected, and help us to determine how best to improve quality of life and functioning of the ever increasing population of childhood cancer survivors.

\section{Acknowledgments}

This project was supported by a grant from the Childhood Cancer Research Fund and evaluations were performed at the General Clinical Research Center of the University of Minnesota (NIH M01-RR00400). The authors have no conflict of interest to declare.

\section{References}

Bennett TL, Ho MR. 1989. The neuropsychology of pediatric epilepsy and antiepileptic drugs. In: Reynolds E ed. Handbook of Clinical Child Neuropsychology: Second Edition. New York, Plenum Press.

Butler R, Mulhern RK. 2005. Neurocognitive interventions for children and adolescents surviving cancer. J Pediatr Psychol, 30:65-78.

Butler RW, Haser JK. 2006. Neurocognitive effects of treatment for childhood cancer. Ment Retard Dev Disabil Res Rev, 12:184-91.

Chan MS, Roebuck DJ, Yuen MP, et al. 2006. MR imaging of the brain in patients cured of acute lymphoblastic leukemia - the value of gradient echo imaging. AJNR Am J Neuroradiol, 27:548-52.

Chan YL, Roebuck DJ, Yuen MP, et al. 2001. Long-term cerebral metabolite changes on proton magnetic resonance spectroscopy in patients cured of acute lymphoblastic leukemia with previous intrathecal methotrexate and cranial irradiation prophylaxis. Int J Radiat Oncol Biol Phys, 50:759-63.
Chu WC, Chik KW, Chan YL, et al. 2003. White matter and cerebral metabolite changes in children undergoing treatment for acute lymphoblastic leukemia: longitudinal study with MR imaging and $1 \mathrm{H}$ MR spectroscopy. Radiology, 229:659-69.

de Groot JC, de Leeuw F, Oudkerk M, et al. (2000). Cerebral White Matter Lesions and Cognitive Function: The Rotterdam Scan Study. Ann Neurol, 147:145-51.

Haykin ME, Gorman M, van Hoff J, et al. 2006. Diffusion-weighted MRI correlates of subacute methotrexate-related neurotoxicity. J Neurooncol, 76:153-7.

Khong PL, Leung LH, Fung AS, et al. 2006. White matter anisotropy in post-treatment childhood cancer survivors: preliminary evidence of association with neurocognitive function. $J$ Clin Oncol, 24:884-90.

Kolb B, Wishaw I. 2003. Fundamentals of Human Neuropsychology: Fourth Edition., W. H. Freeman and Company.

Leiper AD. 2002. Non-endocrine late complications of bone marrow transplantation in childhood: Part 1. Br J Hematol, 118:3-22.

Leung L, Ooi G, Kwong D, et al. 2004. White matter anisotropy after chemo-irradiation: a statistical parametric mapping study and histogram analysis. Neuroimage, 21:261-8.

Lezak M. 1995. Neuropsychological Assessment: Third Edition. New York, Oxford University Press.

Moore B. 2005. Neurocognitive outcomes in survivors of childhood cancer. $J$ Pediatr Psychol, 30:51-63.

Mulhern RK, Palmer SL, Reddick WE, et al. 2001. Risks of young age for selected neurocognitive deficits in medulloblastoma are associated with white matter loss. J Clin Oncol, 19:472-9.

Mulhern RK, Reddick WE, Palmer SL, et al. 1999. Neurocognitive deficits in medulloblastoma survivors and white matter loss. Ann Neurol, 46:834-41.

Paako E, Lehtinen LS, Harila-Saari A, et al. 2003. Perfusion MRI and SPECT of brain after treatment for childhood acute lymphoblastic leukemia. Med Pediatr Oncol, 40:88-92.

Page MP. 2006. What can't functional neuroimaging tell the cognitive psychologist? Cortex, 42:428-43.

Perkins JL, Kunin-Batson AS, Youngren NM, et al. 2007. Long-term follow-up of children who underwent hematopoeitic cell transplant (HCT) for AML or ALL at less than 3 years of age. Pediatr Blood Cancer, 49:958-63

Phipps S, Brenner M, Heslop H, et al. 1995. Psychological effects of bone marrow transplantation on children and adolescents: preliminary report of a longitudinal study. Bone Marrow Transplant, 15:829-35.

Reddick WE, Russell JM, Glass JO, et al. 2000. Subtle white matter volume differences in children treated for medduloblastoma with conventional or reduced dose craniospinal irradiation. Magnetic Resonance Imaging, 18:787-93.

Reddick WE, Shan ZY, Glass JO, et al. 2006. Smaller white-matter volumes are associated with larger deficits in attention and learning among long-term survivors of acute lymphoblastic leukemia. Cancer, 106:941-9.

Ryan C, Geckle MO, Orchard TJ. 2003. Cognitive efficiency declines over time in adults with type 1 diabetes: effects of micro- and macro-vascular complications. Diabtologia, 46:940-8. 
Shapiro EBM. 2000. Metabolic and neurodegenerative disorders. In: Yeates KO, Taylor HG eds. Pediatric Neuropsychology: Research Theory, and Practice. New York, Guilford Press.

Waber D, Mullenix P. 2000. Acute lymphoblastic leukemia. In: Yeates KO, Taylor HG eds. Pediatric Neuropsychology: Research Theory and Practice. New York, Guilford Press.

Waber DP, Carpentieri SC, Klar N, et al. 2000. Cognitive sequelae in children treated for acute lymphoblastic leukemia with dexamethasone or prednisone. J Pediatr Hematol Oncol, 22:206-13.

Wilson DA, Nitschke R, Bowman ME, et al. 1991. Transient white matter changes on MR images in children undergoing chemotherapy for acute lymphocytic leukemia: Correlation with neuropsychologic deficiencies. Radiology, 180:205-9. 